\title{
The Impact of Parity on Course of Labor in a Contemporary Population
}

\author{
Anjel Vahratian, $P h D, M P H$, Matthew K. Hoffman, $M D, M P H$, James F. Troendle, $P h D$, and \\ Jun Zhang, PhD, MD
}

\begin{abstract}
Background: Few studies have examined in depth the labor progression of multiparas to determine if there is any additional impact of being parous beyond the first birth. The objective of this study was to determine the effect of parity on labor progression in contemporary obstetric practice. Methods: Our sample consisted of all low-risk women who delivered a term, live-born infant from January 2002 to March 2004 at a single institution in Delaware, United States $(\mathrm{n}=5,589)$. The median duration of labor by each centimeter of cervical dilation was computed for parity $=0(\mathrm{n}=2,645)$; parity $=1(\mathrm{n}=1,839)$; parity $=2(\mathrm{n}=750)$; and parity $=3+(\mathrm{n}=355)$. Results: Multiparas had a significantly faster labor progression from 4 to $10 \mathrm{~cm}$ (293, 300, and $313 \mathrm{~min}$, respectively, for parity $=1$, parity $=2$, and parity $=3+)$, compared with nulliparas $(383$ min for parity $=0)$, as well as a shorter second stage of labor. However, no significant differences were found in duration of the active phase or the second stage of labor among multiparas. Conclusions: Additional childbearing appears to have no effect of on the progression of labor among multiparous subgroups. The difference in duration of the active phase between nulliparas and multiparas is substantially smaller in a contemporary population. (BIRTH 33:1 March 2006)
\end{abstract}

In the 1950s, Emanuel Friedman examined differences in the progression of labor between nulliparous and multiparous women in a series of publications (1-3). Briefly, he showed that nulliparas progress more slowly throughout both first and second stages

Anjel Vahratian, James Troendle, and Jun Zhang are in the Division of Epidemiology, Statistics, and Prevention Research, National Institute of Child Health and Human Development, National Institutes of Health, Department of Health and Human Services, Bethesda, Maryland; and Matthew Hoffman is in the Department of Obstetrics and Gynecology, Christiana Care Health System, Newark, Delaware, USA. Dr. Vahratian is now affiliated with the University of Michigan, Ann Arbor, Michigan, USA.

This paper was presented in part at the 52nd Annual Clinical Meeting of the American College of Obstetricians and Gynecologists, Philadelphia, Pennsylvania, USA, May 1-5, 2004.

Address correspondence to Dr. Anjel Vahratian, Department of Obstetrics and Gynecology, University of Michigan, L4000 Women's Hospital, 1500 E. Medical Center Drive, Ann Arbor, Michigan 48109-0276, USA.

(C) 2006, Copyright the Authors

Journal compilation (C) 2006, Blackwell Publishing, Inc. of labor compared with multiparas. Moreover, the latent phase of labor appeared to shorten slightly with additional childbearing (3). Subsequent work by Friedman and others over the next three decades confirmed these findings (4-11). These trends are partly due to lessened uterocervical resistance and increased uterine efficiency among multiparas (12).

However, few studies have examined in depth the labor progression of multiparas to determine if being parous beyond the first birth has any additional impact $(6,10,11,13)$. Furthermore, obstetric practice has changed substantially in the past 50 years. Induction of labor, use of oxytocin, epidural analgesia, and cesarean delivery are commonplace, whereas use of forceps, especially at the higher station, is rare (14). Thus, we sought to examine the effect of parity on labor progression in contemporary obstetric practice.

\section{Methods}

This study is a retrospective analysis of obstetric data from the Christiana Hospital in Newark, Delaware, a tertiary care teaching hospital that serves residents of 
the state of Delaware and in neighboring regions of Pennsylvania, Maryland, and New Jersey. In 1998 this institution established an electronic obstetric records system. This database consists of prospectively collected labor and delivery information and manually abstracted information from the prenatal record.

When a woman is admitted to the labor and delivery unit, the nursing staff creates an entry for her in the database. Information such as obstetric interventions performed as well as the details of each vaginal examination, including the time of the exam and cervical dilation, are noted in real-time. Full-time trained abstractors extract and input the information from the prenatal and neonatal record within a month after the delivery. External validation of this database is performed every 3 to 4 months, and the information has been shown to be 95 percent accurate.

Between January 2002 and March 2004, 9,447 women with complete parity information delivered a singleton infant at this institution, of whom 7,657 delivered at term. Further excluded from this analysis were 2,068 women with one or more of the following maternal or fetal conditions during pregnancy or at the time of admission: diabetes; hypertension; maternal medical history of cardiovascular, infectious, pulmonary, renal, mental, or thyroid disorders; multiple gestation; intrauterine growth restriction; uterine bleeding; oligohydramnios; prior cesarean delivery; breech presentation; elective cesarean delivery without a trial of labor; or clinically indicated induction. Thus, our final sample consisted of 5,589 low-risk women with a singleton vertex term pregnancy. The study protocol was reviewed and approved by the institutional review board at Christiana Care Health System.

Parity was constructed as a four-level ordinal categorical variable with the following distribution: nulliparas, parity $=0(n=2,645)$; parity $=1(n=1,839)$; parity $=2(n=750)$; and parity $=3+(n=355)$. The median duration of labor by each centimeter of cervical dilation was computed based on data from serial vaginal examinations and used as a measurement of labor progression, our primary outcome of interest. In this analysis the starting point of labor was at vaginal examination at admission for those in spontaneous labor or at the start of oxytocin infusion for those with an induced labor.

Because many multiparas were not admitted until cervical dilation at $4 \mathrm{~cm}$, our analysis only focused on the duration of labor from 4 to $10 \mathrm{~cm}$ (active phase) and the second stage of labor. To adjust for potential confounders, we also included information on mother's age, race, patient payment status (resident vs private), labor induction, oxytocin use, use and timing of epidural analgesia placement, infant birthweight (representing fetal size), and gestational age at delivery in final models.

\section{Data Analysis}

To assess the effect of parity on labor progression, we first compared various baseline characteristics of the participants, stratified by parity. For continuous variables, such as maternal age, gestational age at delivery, and infant birthweight, mean and standard deviation values were calculated. For continuous variables, such as cervical dilation at admission, the median and the values at the 10th and 90th percentiles were calculated. For categorical variables, percentages were calculated. The Kruskal-Wallis test, a nonparametric comparison of ranks, was used to test differences in distributions among the parity groups.

Next, we used survival analysis, a technique for analyzing time-to-event data, to quantify the duration of the first stage of labor progression and, specifically, the median time elapsed for women to proceed from $1 \mathrm{~cm}$ of cervical dilation to the next for each group. Because continuous monitoring of cervical dilation was not performed and women were admitted into labor at different degrees of cervical dilation, it was impossible to know exactly when an individual first reached a given level of dilation. However, by considering these times as censored, the median duration of labor could be estimated (15).

For each interval of cervical dilation (e.g., from 3 to $4 \mathrm{~cm}$ ), a lower and upper possible time range was computed from each labor log. Thus, each individual contributed an interval-censored value at a given level of dilation. Interval-censoring can be defined as "when the time of event occurrence is known to be somewhere between times a and b, but we don't know exactly when" (16, p 97). For example, if we knew that an individual labor took at least 2 hours to progress from 4 to $5 \mathrm{~cm}$ but no longer than 5 hours, the interval $[2,5]$ would be used to model the underlying distribution of the duration of labor from 4 to $5 \mathrm{~cm}$. It is well established that the duration of labor has a skewed distribution leaning toward the left (i.e., some labors produce a long right tail of the distribution). This distribution generally fits a log normal distribution. Thus, a natural assumption for the data comprising the time interval is that they are log normally distributed, which was consistent with our data.

We fitted a model with a log normal distribution and interval-censored time-to-event data to assess the 
median duration of time elapsed in hours for each centimeter of cervical dilation during labor using the LIFEREG procedure in SAS (17). Adjustment was made for baseline characteristics that were associated with parity and the duration of labor, based on a $p$ value $<0.20$. These covariates included maternal age (continuous), race (white vs nonwhite), labor induction, oxytocin use, timing and use of epidural analgesia, gestational age at delivery, and fetal size. Median traverse times for each centimeter interval in each group were then estimated numerically by finding the time for which the average fitted probability of the event equaled 0.5. Similar methods were used to estimate the median traverse time for the interval from 4 to $10 \mathrm{~cm}$.

\section{Results}

Table 1 presents the baseline sociodemographic and intrapartum characteristics of the overall study population, stratified by parity. Higher parity multiparas (parity $=3+$ ) were slightly older and more often nonwhite, compared with those in the parity $=0$, parity $=1$, and parity $=2$ subgroups. With respect to obstetric interventions, approximately 30 percent of women in the sample received oxytocin for labor induction. The use of oxytocin for labor augmentation and epidural analgesia for pain relief both declined with increasing parity. Nulliparas were more likely to deliver at a later gestation compared with multiparas. Infants in the parity $=1$ and parity $=2$ subgroups were slightly heavier than infants of parity $=0$ and parity $=3+$ subgroups. Eighteen percent of nulliparas in this sample delivered by cesarean section, of which most were performed during the first stage of labor and based on an indication of dystocia.

The unadjusted median duration of labor from 4 to $10 \mathrm{~cm}$ cervical dilation for women in the parity $=0$, parity $=1$, parity $=2$, and parity $=$ 3 + subgroups was 5.67, 4.93, 4.82, and 5.13 hours, respectively. After adjusting for potential confounders, women in the parity $=1$, parity $=2$, and parity $=$ $3+$ subgroups had a significantly faster median duration of labor from 4 to $10 \mathrm{~cm}$, compared with nulliparous women $(4.88,5.00$, and $5.21 \mathrm{hr}$ vs $6.38 \mathrm{hr}$, respectively, each $p<0.01$ ) (Table 2). Minimal change in the median estimates occurred after adjustment for sociodemographic, obstetric, and infant factors.

Multiparas also had a shorter second stage of labor compared with nulliparas. Being parous appears to have no additional impact on labor progression beyond the first birth, however. In a subsequent analysis with parity $=1$ as the referent population, no significant differences were found among the three multiparous subgroups in the median duration of labor from 4 to $10 \mathrm{~cm}$ cervical dilation (data not shown).

\section{Discussion and Conclusions}

Friedman's original 1950s graphical analysis of 500 labors showed that the mean latent and active phases of labor were shorter in multiparas $(5.3$ and $2.2 \mathrm{hr}$, respectively) compared with nulliparas (8.6 and $4.9 \mathrm{hr}$, respectively) $(2,3)$. Friedman further reported that the latent phase of the first stage of labor appeared to shorten slightly with succeeding pregnancies, although such changes were not statistically significant (3). However, the duration of the active phase of first and second stages of labor remained constant with succeeding pregnancies (3).

A decade later, Friedman developed a computer program to derive more accurate estimates of labor duration, and reported a similar trend as that in his earlier work, except for a shorter mean latent phase for both nulliparous $(6.4 \mathrm{hr})$ and multiparous $(4.8 \mathrm{hr})$ women (6). Moreover, the duration of active phase among the latter was about half that of the former in both Friedman's graphical $(2.2 \mathrm{hr}$ vs $4.9 \mathrm{hr})$ and computer-assisted (2.4 hr vs $4.6 \mathrm{hr}$ ) estimates (6).

Gurewitsch et al compared average first-stage labor curves for nulliparous, lower parity multiparous (parity $1-4$ ), and grand multiparous (parity $\geq 5$ ) women (10). Similarly to Friedman's earlier publications, the authors showed that the average labor curve of lower parity multiparas was distinctly different from the average labor curve of nulliparas. In contrast, the average labor curve of grand multiparas had a similar latent phase as that of nulliparas. However, once grand multiparas transitioned into the active phase of labor, their average labor curve reflected that of lower parity multiparas.

In an analysis of 2,242 women with a spontaneous onset of labor, Bergsjo et al reported that the median duration of the latent (onset of labor to $4 \mathrm{~cm}$ dilation) and active (4 to $10 \mathrm{~cm}$ ) phases of the first stage of labor declined with increasing parity (4). However, their median estimates from 4 to $10 \mathrm{~cm}$ dilation for each parity subgroup ( $2 \mathrm{hr} 26 \mathrm{~min}$ for nulliparas; $1 \mathrm{hr} 45 \mathrm{~min}$ for women with a parity $=1$; and $1 \mathrm{hr} 26 \mathrm{~min}$ for women with a parity $\geq 2$ ) are shorter than those identified in our analysis. Likewise, Juntunen and Kirkinen also showed in 126 women with spontaneous onset of labor that the mean duration of the latent (onset of labor to $4 \mathrm{~cm}$ dilation) and active (4 to $10 \mathrm{~cm}$ ) phases of the first stage of labor declined with increasing parity 


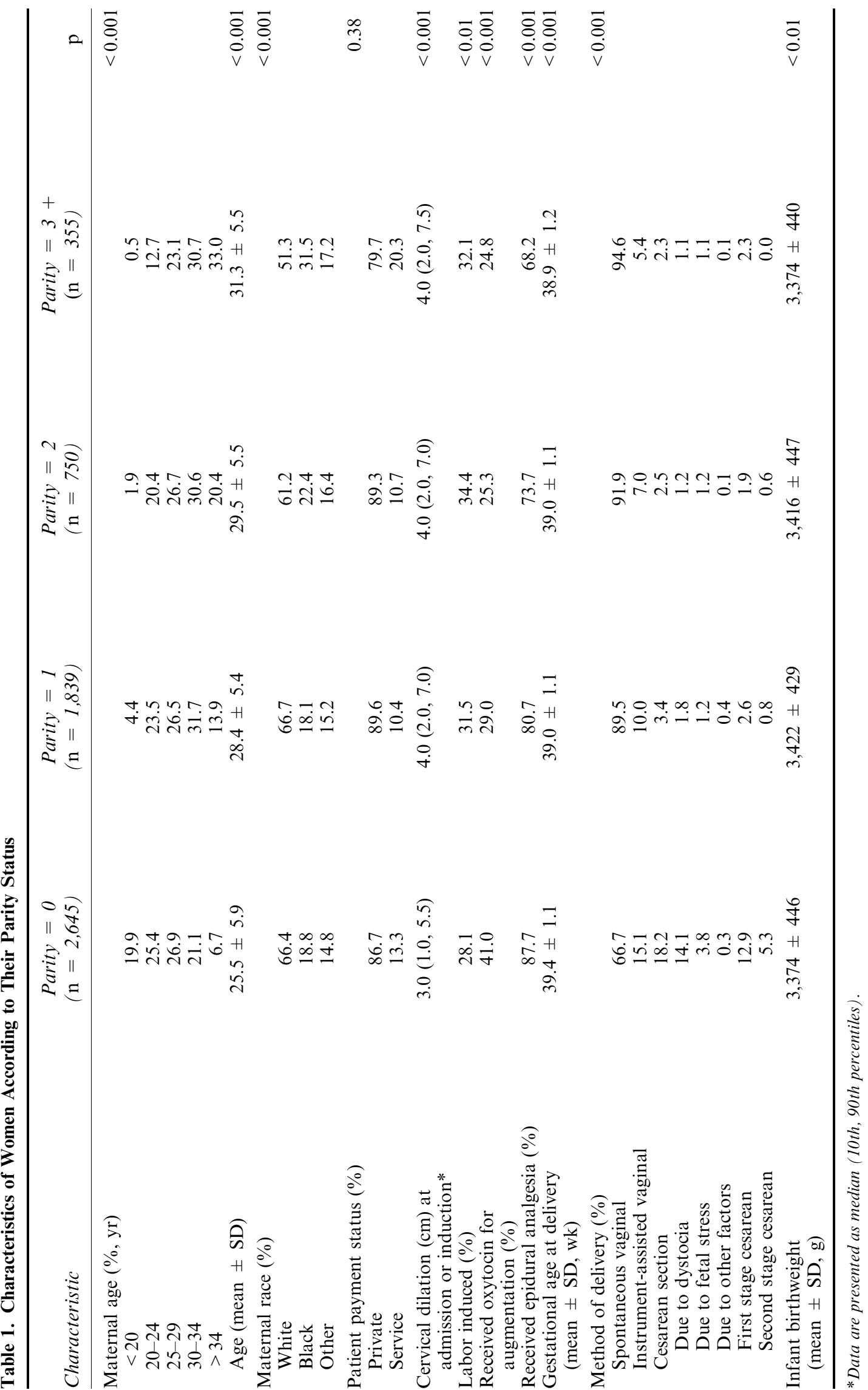


(13). Similarly to Bergsjo et al, their estimates of active phase duration were shorter $(3.1 \mathrm{hr}$ for nulliparas; $2.7 \mathrm{hr}$ for multiparas; and $2.8 \mathrm{hr}$ for grand multiparas) than our findings.

Comparing estimates of duration of labor across studies, especially for the first stage, is difficult for several reasons. First, studies often use different starting points to calculate the duration of labor. Most literature on labor progression before 1990 defined the onset of labor as the presence of regular uterine contractions, which was often based on maternal selfreport if the woman was admitted in spontaneous labor $(4,5,7,8,13,18,19)$. In comparison, recent studies measured duration of the first stage of labor from 4 to $10 \mathrm{~cm}$ dilation, since few spontaneously laboring women are admitted to labor and delivery before $4 \mathrm{~cm}$ dilation in current obstetric practice $(9,20)$. As a result, it is difficult to calculate the duration of the latent phase of labor accurately for the complete study population. Both the measurement and interpretation of the duration of labor is likely to vary greatly based on the selected starting point.

Second, studies on labor progression often restrict their sample populations differently. For example, some studies included only women with a spontaneous onset of labor $(4,7,9-11,13)$, whereas others also included those with an induced labor $(2,3,6,18)$. In addition, some studies further excluded women who delivered by cesarean section $(7,9,13,18)$. Exclusion of induced labor and cesarean deliveries tends to produce shorter durations of labor.

Third, statistical methods used to compute duration of labor may also have made important differences. For instance, the median duration is a better statistic than mean duration, since the distribution of labor duration often has a long right tail. Thus, the mean is often skewed by long labors and shows a longer average labor than the median.

Fourth, using duration of labor from 4 to $10 \mathrm{~cm}$ dilation as a more objective measure of the active phase of labor also can be problematic. For example, women may be admitted to labor and delivery after $4 \mathrm{~cm}$ of cervical dilation, a common issue for multiparas. Moreover, some women may not have an observed cervical exam measurement at $4 \mathrm{~cm}$. Women who proceed to a cesarean delivery before reaching $10 \mathrm{~cm}$ dilation would likely be omitted from the summary measure, since they will not have a recorded measurement at $10 \mathrm{~cm}$. In sum, the fluidity of the population in labor makes broad-based summary measures of labor progression difficult to calculate and generalize to other populations.

Our study population includes spontaneous as well as induced labor, to better reflect contemporary obstetric practice. Furthermore, our statistical method takes into account women who arrived at the hospital after $4 \mathrm{~cm}$ dilation or in whom the observation of $4 \mathrm{~cm}$ was missed and partial information was contributed by first-stage cesarean deliveries. We also adjusted for maternal baseline characteristics and intrapartum procedures. Although the duration of the active phase may still be somewhat shorter than anticipated due to informative censoring of the firststage cesarean deliveries (because cesarean deliveries tend to have a slower labor), our method has made substantial improvement in these aspects.

Our study found that the difference in duration of labor between nulliparas and multiparas is much

Table 2. Median Duration of Labor Progression (in Hours), According to Parity

\begin{tabular}{|c|c|c|c|c|c|c|c|}
\hline Cervical Dilation & $\begin{array}{l}\text { Parity }=0 \\
(\mathrm{n}=2,645)\end{array}$ & $\begin{array}{l}\text { Parity }=1 \\
(\mathrm{n}=1,839)\end{array}$ & $\mathrm{p}^{\dagger}$ & $\begin{array}{l}\text { Parity }=2 \\
(\mathrm{n}=750)\end{array}$ & $\mathrm{p} t$ & $\begin{array}{c}\text { Parity }=3+ \\
\quad(\mathrm{n}=355)\end{array}$ & $\mathrm{p} \S$ \\
\hline $\begin{array}{l}\text { Unadjusted } \\
\text { From } 4 \text { to } 10 \mathrm{~cm}\end{array}$ & 5.67 & 4.93 & $<0.001$ & 4.82 & 0.001 & 5.13 & 0.17 \\
\hline $\begin{array}{l}\text { Adjusted* } \\
\text { From } 4 \text { to } 10 \mathrm{~cm} \\
\text { From } 4 \text { to } 5 \mathrm{~cm} \\
\text { From } 5 \text { to } 6 \mathrm{~cm} \\
\text { From } 6 \text { to } 7 \mathrm{~cm} \\
\text { From } 7 \text { to } 8 \mathrm{~cm} \\
\text { From } 8 \text { to } 9 \mathrm{~cm} \\
\text { From } 9 \text { to } 10 \mathrm{~cm}\end{array}$ & $\begin{array}{l}6.38 \\
1.14 \\
0.73 \\
0.55 \\
0.43 \\
0.41 \\
0.41\end{array}$ & $\begin{array}{l}4.88 \\
0.96 \\
0.61 \\
0.44 \\
0.34 \\
0.30 \\
0.28\end{array}$ & $\begin{array}{l}<0.001 \\
<0.01 \\
<0.001 \\
<0.001 \\
<0.001 \\
<0.001 \\
<0.001\end{array}$ & $\begin{array}{l}5.00 \\
1.00 \\
0.62 \\
0.44 \\
0.33 \\
0.29 \\
0.27\end{array}$ & $\begin{aligned}< & 0.001 \\
& 0.09 \\
& 0.01 \\
< & 0.001 \\
< & 0.001 \\
< & 0.001 \\
< & 0.001\end{aligned}$ & $\begin{array}{l}5.21 \\
1.06 \\
0.59 \\
0.45 \\
0.32 \\
0.30 \\
0.27\end{array}$ & $\begin{aligned}< & 0.01 \\
& 0.51 \\
& 0.02 \\
& 0.01 \\
< & 0.001 \\
< & 0.001 \\
< & 0.001\end{aligned}$ \\
\hline $\begin{array}{l}\text { Second stage of labor } \\
\quad \text { (geometric mean, minutes) }\end{array}$ & 51 & 17 & $<0.001$ & 12 & $<0.001$ & 10 & $<0.001$ \\
\hline
\end{tabular}

*Adjusted for maternal age, maternal race, labor induction, oxytocin use, epidural analgesia, timing of epidural analgesia placement, infant birthweight, and gestational age at delivery; $\uparrow$ comparison between parity $=1$ and parity $=0$; $\$$ comparison between parity $=2$ and parity $=0$; $\S$ comparison between parity $=3+$ and parity $=0$. 
smaller now than previously reported. This may partly reflect changes in the patient population. For example, the prevalence of obesity, that is, body mass index (BMI $\geq 30 \mathrm{~kg} / \mathrm{m}^{2}$ ), and extreme obesity (BMI $\geq 40 \mathrm{~kg} / \mathrm{m}^{2}$ ) is rising among women of reproductive age (21). Vahratian et al recently showed that nulliparas who are obese experience a slower first and second stage of labor, compared with normal weight women (22). Since multiparas in general have a higher prevalence of overweight and obesity than nulliparas (23-26), it is reasonable to infer that their labor may be prolonged with increasing BMI as well. Unfortunately, this database did not routinely document maternal pre-pregnancy weight and height to calculate BMI; thus, we were unable to test this hypothesis in the present study.

An inherent limitation of our study is that the measurement of cervical dilation was subjective. Continuous monitoring of cervical dilation was not performed, and the measurements were based on vaginal examinations performed by several physicians. This potential misclassification would likely be nondifferential in nature and would bias our results toward the null. In addition, the median cervical dilation at admission or at the time of induction in our study population was $3.0 \mathrm{~cm}$ in nulliparas and $4.0 \mathrm{~cm}$ in multiparas. The small number of observations at 1 to $3 \mathrm{~cm}$ precluded us from examining parityspecific differences during the latent phase of labor. Our sample population was drawn from one tertiary care hospital in Delaware, which also may limit the generalizability of our results.

In conclusion, our data indicate that multiparas have a faster labor progression from 4 to $10 \mathrm{~cm}$ dilation and in the second stage of labor when compared with nulliparas. However, the difference in the duration of active phase of labor among nulliparous and multiparous women is smaller in contemporary obstetric practice. Moreover, additional childbearing had no effect on the progression of labor among multiparas beyond the first delivery.

\section{References}

1. Friedman EA. The graphic analysis of labor. Am J Obstet Gynecol 1954;68:1568-1575.

2. Friedman EA. Primigravid labor: A graphicostatistical analysis. Obstet Gynecol 1955;6:567-589.

3. Friedman EA. Labor in multiparas: A graphicostatistical analysis. Obstet Gynecol 1956;8:691-703.
4. Bergsjo P, Bakketeig L, Eikhom SN. Duration of labour with spontaneous onset. Acta Obstet Gynecol Scand 1979;58: 129-134.

5. Friedman EA. Labor: Clinical Evaluation \& Management. 2nd ed. New York: Appleton-Century-Crofts, 1978.

6. Friedman EA, Kroll BH. Computer analysis of labor progression. III. Pattern variations by parity. J Reprod Med 1971;6:179-183.

7. Kilpatrick SJ, Laros Jr RK. Characteristics of normal labor. Obstet Gynecol 1989;74:85-87.

8. Peisner DB, Rosen MG. Latent phase of labor in normal patients: A reassessment. Obstet Gynecol 1985;66:644-648.

9. Albers LL, for the CNM Data Group 1996. The duration of labor in healthy women. J Perinatol 1999;19:114-119.

10. Gurewitsch ED, Diament P, Fong J, et al. The labor curve of the grand multipara: Does progress of labor continue to improve with additional childbearing? Am J Obstet Gynecol 2002;186:1331-1338.

11. Gurewitsch ED, Johnson E, Allen RH, et al. The descent curve of the grand multiparous woman. Am J Obstet Gynecol 2003;189:1036-1041.

12. Arulkumaran S, Gibb DMF, Lun KC, et al. The effect of parity on uterine activity in labour. Br J Obstet Gynaecol 1984;91:843-848.

13. Juntunen K, Kirkinen P. Partogram of a grand multipara: Different descent slope compared with an ordinary parturient. J Perinat Med 1994;22:213-218.

14. Martin JA, Hamilton BE, Sutton PD, et al. Births: Final data for 2002. Natl Vital Stat Rep 2003;52(10):1-27.

15. Klein JP, Moeschberger ML. Survival Analysis: Techniques for Censored and Truncated Data. Berlin: Springer, 1997.

16. Allison PD. Survival Analysis Using the SAS System: A Practical Guide: SAS Institute Inc., 1995.

17. SAS Institute Inc. SAS Online Doc, Version 8, Cary, NC: SAS Institute Inc., 1999.

18. Nesheim B. Duration of labor: An analysis of influencing factors. Acta Obstet Gynecol Scand 1988;67:121-124.

19. Friedman EA. The labor curve. Clin Perinatol 1981;8:15-25.

20. Albers LL, Schiff M, Gorwoda JG. The length of active labor in normal pregnancies. Obstet Gynecol 1996;87:355-359.

21. Hedley AA, Ogden CL, Johnson CL, et al. Prevalence of overweight and obesity among US children, adolescents, and adults, 1999-2002. JAMA 2004;291:2847-2850.

22. Vahratian A, Zhang J, Troendle JF, Savitz DA, Siega-Riz AM. Maternal pre-pregnancy overweight and obesity and the pattern of labor progression in term nulliparous women. Obstet Gynecol 2004;104:943-951.

23. Gunderson E, Abrams B. Epidemiology of gestational weight gain and body weight changes after pregnancy. Epidemiol Rev 1999;21:261-275.

24. Lederman SA. The effect of pregnancy weight gain on later obesity. Obstet Gynecol 1993;82:148-155.

25. Rosenberg L, Palmer JR, Wise LA, et al. A prospective study of the effect of childbearing on weight gain in AfricanAmerican women. Obes Res 2003;11:1526-1535.

26. Siega-Riz AM, Evenson KR, Dole N. Pregnancyrelated weight gain - a link to obesity? Nutr Rev 2004;62:S105-S111. 